\title{
Maternal gestational diabetes and childhood obesity at age 9-11: results of a multinational study
}

\author{
Pei Zhao ${ }^{1}$ Enqing Liu ${ }^{1}$ - Yijuan Qiao ${ }^{1,2}$ - Peter T. Katzmarzyk ${ }^{2}$. \\ Jean-Philippe Chaput ${ }^{3} \cdot$ Mikael Fogelholm $^{4}$ - William D. Johnson ${ }^{2} \cdot$ Rebecca Kuriyan $^{5}$. \\ Anura Kurpad $^{5}$ - Estelle V. Lambert ${ }^{6} \cdot$ Carol Maher $^{7}$ - José A.R. Maia ${ }^{8}$. \\ Victor Matsudo ${ }^{9}$. Timothy Olds ${ }^{7}$. Vincent Onywera ${ }^{10}$ • Olga L. Sarmiento ${ }^{11}$. \\ Martyn Standage ${ }^{12}$ - Mark S. Tremblay ${ }^{3}$ - Catrine Tudor-Locke ${ }^{2,13}$ - Gang $\mathrm{Hu}^{2}$ • for the \\ ISCOLE Research Group
}

Received: 15 March 2016 / Accepted: 1 July 2016 / Published online: 11 August 2016

(C) Springer-Verlag Berlin Heidelberg 2016

\begin{abstract}
Aims/hypothesis The aim of this study was to examine the association between maternal gestational diabetes mellitus (GDM) and childhood obesity at age 9-11 years in 12 countries around the world.

Methods A multinational cross-sectional study of 4740 children aged 9-11 years was conducted. Maternal GDM was diagnosed according to the ADA or WHO criteria. Height and waist circumference were measured using standardised methods. Weight and body fat were measured using a portable Tanita SC-240 Body Composition Analyzer. Multilevel
\end{abstract}

A list of members of the ISCOLE Research Group is given in the electronic supplementary material (ESM).

Electronic supplementary material The online version of this article (doi:10.1007/s00125-016-4062-9) contains peer-reviewed but unedited supplementary material, which is available to authorised users.

Gang $\mathrm{Hu}$

gang.hu@pbrc.edu

1 Tianjin Women's and Children's Health Center, Tianjin, China

2 Pennington Biomedical Research Center, 6400 Perkins Road, Baton Rouge, LA 70808, USA

3 Children's Hospital of Eastern Ontario Research Institute, Ottawa, ON, Canada

4 Department of Food and Environmental Sciences, University of Helsinki, Helsinki, Finland

5 St Johns Research Institute, Bangalore, India

6 Division of Exercise Science and Sports Medicine, Department of Human Biology, Faculty of Health Sciences, University of Cape Town, Cape Town, South Africa modelling was used to account for the nested nature of the data.

Results The prevalence of reported maternal GDM was $4.3 \%$. The overall prevalence of childhood obesity, central obesity and high body fat were $12.3 \%, 9.9 \%$ and $8.1 \%$, respectively. The multivariable-adjusted (maternal age at delivery, education, infant feeding mode, gestational age, number of younger siblings, child unhealthy diet pattern scores, moderate-tovigorous physical activity, sleeping time, sedentary time, sex and birthweight) odds ratios among children of GDM mothers compared with children of non-GDM mothers were 1.53

7 Alliance for Research in Exercise Nutrition and Activity (ARENA), School of Health Sciences, University of South Australia, Adelaide, Australia

$8 \mathrm{CIFI}^{2} \mathrm{D}$, Faculdade de Desporto, University of Porto, Porto, Portugal

9 Centro de Estudos do Laboratório de Aptidão Física de São Caetano do Sul, São Paulo, Brazil

10 Department of Recreation Management and Exercise Science, Kenyatta University, Nairobi, Kenya

11 School of Medicine, Universidad de los Andes, Bogotá, Colombia

12 Department for Health, University of Bath, Bath, UK

13 Department of Kinesiology, University of Massachusetts Amherst, Amherst, USA 
(95\% CI $1.03,2.27)$ for obesity, 1.73 (95\% CI 1.14, 2.62) for central obesity and 1.42 (95\% CI 0.90, 2.26) for high body fat. The positive association was still statistically significant for central obesity after additional adjustment for current maternal BMI but was no longer significant for obesity and high body fat.

Conclusions/interpretation Maternal GDM was associated with increased odds of childhood obesity at 9-11 years old but this association was not fully independent of maternal BMI.

Keywords Children · Gestational diabetes · Obesity

$\begin{array}{ll}\text { Abbreviations } \\ \text { FFQ } & \text { Food frequency questionnaire } \\ \text { GDM } & \text { Gestational diabetes mellitus } \\ \text { ISCOLE } & \begin{array}{l}\text { International Study of Childhood Obesity, } \\ \text { Lifestyle and the Environment }\end{array} \\ \text { NHANES } & \begin{array}{l}\text { National Health and Nutrition Examination } \\ \text { Survey }\end{array}\end{array}$

\section{Introduction}

Childhood obesity has increased dramatically in both developed and developing countries [1]. It has been suggested that prenatal, perinatal and postnatal environmental factors impact childhood obesity [2]. Some studies have found that intrauterine exposure to maternal gestational diabetes mellitus (GDM) places offspring at increased risk of long-term adverse outcomes, including obesity [3-12]. GDM, defined as any degree of glucose intolerance with onset or first recognition during pregnancy [3], is a common pregnancy complication affecting approximately 1-28\% of pregnancies in a survey of 173 countries, based on uniform diagnostic criteria for GDM [4].

Early research from the Pima Indian Study and the Diabetes in Pregnancy Study at Northwestern University in the USA provided initial evidence of an association between maternal GDM and the risk of childhood obesity [5, 6]. However, other studies failed to find a clear association between maternal GDM and offspring obesity [7-12]. A recent literature review indicated that this difference may be due to the high type 2 diabetes mellitus risk in the unique Pima Indian population and the specialised clinical population in the Northwestern University study [2]. Furthermore, the majority of the previous studies are from high income countries, with limited data from low to middle income countries; thus studies that include children from multiple regions of the world are needed.

Indicators of central obesity, such as waist circumference, may better predict cardiovascular disease compared with adiposity measured by BMI [13]. However, limited data exist on the association between maternal GDM and different indicators of childhood obesity. The aim of the present study was to examine the association between self-reported maternal GDM and three indicators of childhood obesity (BMI, waist circumference and body fat) in children aged 9-11 years from 12 countries around the world.

\section{Methods}

\section{Study design}

The International Study of Childhood Obesity, Lifestyle and the Environment (ISCOLE) is a multinational cross-sectional study conducted at urban and suburban sites in 12 countries (Australia, Brazil, Canada, China, Colombia, Finland, India, Kenya, Portugal, South Africa, the UK and the USA) [14]. These countries are classified as low to high income countries according to the World Bank Classification (Table 1). More details on the study design and methods can be found elsewhere [14]. The Institutional Review Board at the Pennington Biomedical Research Center (coordinating centre) approved the overarching protocol, and the institutional/ethical review boards at each participating institution approved the local protocols. Written informed consent was obtained from parents or legal guardians, and child assent was also obtained as required by the local institutional/ethical review boards before participation in the study.

\section{Participants}

A total of 7372 children aged 9-11 years participated in ISCOLE. Of these children, 4740 remained after excluding participants who did not have valid data/information on accelerometer $(n=1214)$, maternal history of GDM $(n=359)$, BMI $(n=5)$, waist circumference $(n=4)$, percentage of body fat $(n=58)$, birthweight $(n=383)$, gestational age $(n=101)$, diet scores $(n=82)$, maternal age at child's birth $(n=134)$, maternal current BMI $(n=216)$ and other information (maternal education and infant feeding mode) $(n=76)$. Participants who were excluded did not differ in age or BMI $z$ score but there was a higher proportion of boys compared with those who were included in the analysis. Data were collected from September 2011 to December 2013.

\section{Measurements}

Demographics and family health history Maternal education, current maternal body weight and height, maternal age at child's birth, child age, child sex, birthweight, infant feeding mode, gestational age and number of younger siblings were collected from parents or guardians by a demographic and family health history questionnaire. Maternal education was 
Table 1 Prevalence of GDM and diagnostic criteria used at the 12 study sites, 1999-2004

\begin{tabular}{|c|c|c|c|c|c|}
\hline \multirow[t]{2}{*}{ Country (site) } & \multirow{2}{*}{$\begin{array}{l}\text { World bank } \\
\text { classification }\end{array}$} & \multirow{2}{*}{$\begin{array}{l}\text { Diagnostic criteria } \\
\text { for GDM }\end{array}$} & \multirow{2}{*}{$\begin{array}{l}\text { No. of } \\
\text { participants }\end{array}$} & \multicolumn{2}{|l|}{ GDM } \\
\hline & & & & No. of cases & Prevalence $(\%)$ \\
\hline Australia (Adelaide) & High income & Modified WHO & 386 & 20 & 5.2 \\
\hline Canada (Ottawa) & High income & ADA & 443 & 14 & 3.2 \\
\hline Finland (Helsinki, Espoo and Vantaa) & High income & WHO & 401 & 22 & 5.5 \\
\hline Portugal (Porto) & High income & Modified ADA & 533 & 47 & 8.8 \\
\hline UK (Bath and NE Somerset) & High income & WHO & 324 & 6 & 1.9 \\
\hline USA (Baton Rouge) & High income & $\mathrm{ADA}$ & 363 & 21 & 5.8 \\
\hline Brazil (São Paulo) & Upper-middle income & WHO & 354 & 11 & 3.1 \\
\hline China (Tianjin) & Upper-middle income & WHO & 413 & 8 & 1.9 \\
\hline Colombia (Bogotá) & Upper-middle income & $\mathrm{ADA}$ & 700 & 23 & 3.3 \\
\hline South Africa (Cape Town) & Upper-middle income & WHO & 120 & 6 & 5.0 \\
\hline India (Bangalore) & Lower-middle income & Modified ADA & 414 & 20 & 4.8 \\
\hline Kenya (Nairobi) & Low income & WHO & 289 & 8 & 2.8 \\
\hline All sites & & & 4740 & 206 & 4.3 \\
\hline
\end{tabular}

classified into three categories: did not complete high school, completed high school or some college, and completed bachelor or postgraduate degree. The child's parents were asked whether the child was fed breast milk or not, age when completely stopped being fed breast milk, age when first fed formula and age when completely stopped drinking formula. These responses were classified into four categories: exclusive breast feeding, mixed feeding, weaned from breast feeding and exclusive formula feeding.

Maternal history of GDM Maternal history of GDM was recalled by parents or guardians and reported on the questionnaire. Maternal GDM was diagnosed between July 1999 and July 2004, inferred by the child's date of birth. The diagnostic criteria for GDM used in local maternity hospitals during this period were identified by each study site. Maternal GDM was diagnosed by the WHO or modified WHO criteria based on a $2 \mathrm{hr} 75 \mathrm{~g}$ OGTT, or the ADA or modified ADA criteria based on $3 \mathrm{hr} 100 \mathrm{~g}$ OGTT $[15,16]$. The WHO criteria for GDM requires one plasma glucose test result of $\geq 7.0 \mathrm{mmol} / 1$ (fasting) or $\geq 7.8 \mathrm{mmol} / \mathrm{l}(2 \mathrm{~h})[15]$. The ADA criteria requires two plasma glucose test results of $\geq 5.3 \mathrm{mmol} / 1$ (fasting), $\geq 10.0 \mathrm{mmol} / 1$ $(1 \mathrm{~h}), \geq 8.6 \mathrm{mmol} / \mathrm{l}(2 \mathrm{~h})$ or $\geq 7.8 \mathrm{mmol} / \mathrm{l}(3 \mathrm{~h})[16]$.

Dietary pattern A food frequency questionnaire (FFQ) that was adapted from the Health Behavior in School-aged Children Survey (HBSC) and validated [17-19] was administered to all ISCOLE participants. The FFQ asked the participants about their 'usual' consumption of 23 food categories, with response options including: never, less than once per week, once per week, 2-4 days per week, 5-6 days per week, once a day every day, and more than once a day. Two diet pattern scores that represented an 'unhealthy diet pattern' (with positive loadings for fast food, hamburgers, soft drinks, sweets, fried food, etc.) and a 'healthy diet pattern' (with positive loadings for vegetables, fruit, whole grains, low-fat milk, etc.) were obtained using principal components analyses $[18,19]$.

Anthropometric measurements A battery of anthropometric measurements was taken according to standardised procedures across all study sites. Height was measured without shoes using a Seca 213 portable stadiometer (Seca, Hamburg, Germany), with the participant's head in the Frankfurt plane. Waist circumference was measured at the end of normal expiration with a non-elastic tape held midway between the lower rib margin and the iliac crest. Each measurement was repeated and the average was used for analysis (a third measurement was obtained if the difference between the first two measurements was $>0.5 \mathrm{~cm}$, then the average of the two closest measurements was used in the analyses) [14].

The participant's weight and percentage of body fat were measured using a portable Tanita SC-240 Body Composition Analyzer (Tanita, Arlington Heights, IL). All outer clothing, heavy pocket items, and shoes and socks were removed. Two measurements were obtained and the average was used in the analysis (a third measurement was obtained if the difference between the first two measurements was $>0.5 \mathrm{~kg}$ or $>2.0 \%$ for weight and percentage of body fat, respectively, then the average of the two closest measurements was used in the analyses)[14].

Maternal and childhood BMI were calculated by dividing weight in kilograms by the square of height in metres. Childhood BMI $z$ scores were computed using age- and sexspecific reference data from the WHO. Waist circumference $z$ scores were computed using age- and sex-specific reference data from the National Health and Nutrition Examination Survey III (NHANES III) from 1988 to 1994 [20]. Body fat $z$ scores were 
computed using the NHANES IV data from 1999 to 2004 [21]. Child obesity was defined as BMI $z$ scores $>+2$ SD. Central obesity was defined as waist circumference $\geq 90$ th percentile of NHANES III reference [22]. High body fat was defined as body fat $\geq 90$ th percentile of NHANES IV reference [21]. Maternal overweight was defined as BMI $\geq 25 \mathrm{~kg} / \mathrm{m}^{2}$, based on self-reported maternal current height and weight.

Accelerometry An ActiGraph GT3X+ accelerometer (ActiGraph, Pensacola, FL, USA) was used to objectively measure moderate-to-vigorous physical activity, sedentary behaviour and sleeping time. The accelerometer was worn at the waist on an elasticated belt on the right mid-axillary line. Participants were encouraged to wear the accelerometer $24 \mathrm{~h}$ per day (removing it only for water-related activities) for at least 7 days including two weekend days (plus an initial familiarisation day and the morning of the final day)[14]. Nocturnal sleep duration was estimated from the accelerometer data using a fully automated algorithm for $24 \mathrm{~h}$ waist-worn accelerometers, which was recently validated for ISCOLE [23]. The weekly total sleep time averages were calculated using only days where valid sleep was accumulated (total sleep period time $\geq 160 \mathrm{~min}$ ) and only for participants with at least three nights of valid sleep including one weekend day [24]. After exclusion of total sleep time and awake non-wear time (any sequence of $\geq 20$ consecutive minutes of zero activity counts), moderate-to-vigorous physical activity was defined as all activity $\geq 574$ counts per $15 \mathrm{~s}$ and total sedentary time was defined as all movement $\leq 25$ counts per $15 \mathrm{~s}$, consistent with the widely used Evenson cut-offs [25].

\section{Statistical analysis}

Variables were compared using a $t$ test for means and a $\chi^{2}$ test for proportions between women with and without GDM. Multilevel linear regression models were used to estimate the association between maternal GDM and $z$ scores of childhood BMI, waist circumference and body fat. Multilevel logistic regression models were used to estimate the association between maternal GDM and the odds of childhood obesity, central obesity and high body fat. We defined child as level 1, school as level 2 and study site as level 3 for the multilevel analyses. Study site and school were considered to have random effects. The analyses were adjusted for maternal age at delivery (continuous variable), maternal current BMI (continuous variable), maternal education (categorical variable), infant feeding mode (categorical variable), birthweight (continuous variable), gestational age (continuous variable), number of younger siblings (continuous variable), child unhealthy diet pattern scores (continuous variable), moderate-to-vigorous physical activity (continuous variable), sleeping time (continuous variable), sedentary time (continuous variable), age (continuous variable) and sex (categorical variable). The criterion for statistical significance was $p<0.05$. All statistical analyses were performed with SPSS for Windows, version 21.0 (Statistics 21, SPSS, IBM, USA) or SAS for Windows, version 9.4 (SAS Institute, Cary, NC).

\section{Results}

The prevalence of maternal GDM and the diagnostic criteria employed between 1999 and 2004 at the 12 study sites are presented in Table 1. The overall prevalence of self-reported maternal GDM was $4.3 \%$, ranging from $1.9 \%$ in the UK and China to $8.8 \%$ in Portugal. Characteristics of study participants by maternal GDM status are presented in Table 2 . GDM mothers had significantly older age at delivery than non-GDM mothers (29.9 years vs 28.3 years). Children of GDM mothers had significantly higher mean birthweight (3415 g vs $3274 \mathrm{~g}$ ), and significantly higher prevalence of obesity ( $18.4 \%$ vs $12.0 \%)$, central obesity ( $16.0 \%$ vs $9.6 \%$ ) and high body fat $(12.1 \%$ vs $7.9 \%)$ at age $9-11$ years compared with children of non-GDM mothers.

After adjustment for maternal age at delivery and education, infant feeding mode, gestational age, number of younger siblings, child unhealthy diet pattern scores, moderate-tovigorous physical activity, sleeping time, sedentary time, sex and birthweight, children of GDM mothers had significantly higher mean values for BMI $z$ score $(0.71$ vs 0.54$)$, waist circumference $z$ score $(0.06$ vs -0.02$)$ and body fat $z$ score ( 0.17 vs 0.02 ) than children of non-GDM mothers (Table 3 ). These significant associations disappeared after additional adjustment for current maternal BMI.

Table 4 presents the association of maternal GDM with the risk of childhood obesity, central obesity and high body fat by all GDM mothers or GDM mothers with normal weight or overweight. The multivariable-adjusted odds ratios among children of GDM mothers compared with children of non-GDM mothers were 1.53 (95\% CI 1.03, 2.27) for obesity, 1.73 (95\% CI 1.14, 2.62) for central obesity and $1.42(95 \%$ CI $0.90,2.26)$ for high body fat (Table 4). The positive association between GDM and central obesity was still significant after additional adjustment for current maternal BMI, but not for general obesity or high body fat. In the multivariable-adjusted analyses, the positive association of maternal GDM with the odds of childhood obesity and central obesity were present among GDM mothers who were overweight but not among GDM mothers with normal weight.

When stratified by maternal GDM diagnostic criteria, sex of the child, level of moderate-to-vigorous physical activity, unhealthy diet scores, sleep time, breastfeeding status and the country's income classification, the positive association of maternal GDM with the odds of central obesity was only present in girls and in children of mothers whose GDM was diagnosed by the ADA criteria (Table 5). There were no significant interactions between maternal GDM and diagnostic criteria, the child's sex, level of moderate-to-vigorous physical activity, unhealthy diet pattern scores, sleep time, the 
Table 2 Characteristics of study participants by maternal GDM status

\begin{tabular}{|c|c|c|c|}
\hline Characteristic & Non-GDM $(n=4534)$ & $\operatorname{GDM}(n=206)$ & $p$ value \\
\hline \multicolumn{4}{|l|}{ Maternal characteristics } \\
\hline Age at delivery (years) & $28.3(5.7)$ & $29.9(5.8)$ & $<0.001$ \\
\hline Current BMI $\left(\mathrm{kg} / \mathrm{m}^{2}\right)$ & $25.6(4.9)$ & $27.5(5.0)$ & $<0.001$ \\
\hline Current overweight status, $n(\%)$ & $2083(45.9)$ & $130(63.1)$ & $<0.001$ \\
\hline Education, $n(\%)$ & & & 0.54 \\
\hline Did not complete high school & $997(22.0)$ & $52(25.2)$ & \\
\hline Completed high school/some college & $2064(45.5)$ & $89(43.2)$ & \\
\hline Bachelor's or postgraduate degree & $1473(32.5)$ & $65(31.6)$ & \\
\hline \multicolumn{4}{|l|}{ Offspring characteristics at birth or first year } \\
\hline Boys, $n(\%)$ & $2091(46.1)$ & $95(46.1)$ & 1.00 \\
\hline Birthweight (g) & $3274(576)$ & $3415(623)$ & 0.001 \\
\hline Gestational age (weeks) & $38.6(2.2)$ & $38.3(2.1)$ & 0.035 \\
\hline Infant breast feeding, $n(\%)$ & & & 0.16 \\
\hline Exclusive breast feeding & $1722(38.0)$ & $63(30.6)$ & \\
\hline Mixed feeding & $2137(47.1)$ & $105(51.0)$ & \\
\hline Weaned from breast feeding & $47(1.0)$ & $3(1.5)$ & \\
\hline Exclusive formula feeding & $628(13.9)$ & $35(17.0)$ & \\
\hline \multicolumn{4}{|l|}{ Offspring characteristics at age 9-11 years } \\
\hline Age (years) & $10.4(0.6)$ & $10.4(0.5)$ & 0.76 \\
\hline Younger siblings $^{\mathrm{a}}$ & 0.60 & 0.55 & 0.39 \\
\hline BMI $\left(\mathrm{kg} / \mathrm{m}^{2}\right)$ & $18.4(3.4)$ & $19.1(3.6)$ & 0.002 \\
\hline Waist circumference $(\mathrm{cm})$ & $64.2(8.8)$ & $66.3(9.5)$ & 0.001 \\
\hline Body fat $(\%)$ & $20.8(7.6)$ & $22.4(7.6)$ & 0.002 \\
\hline Unhealthy diet pattern score & $-0.15(0.85)$ & $-0.13(0.93)$ & 0.69 \\
\hline Moderate-to-vigorous physical activity (min/day) & $59.6(24.7)$ & $59.0(24.1)$ & 0.71 \\
\hline Sedentary time (min/day) & $518(68)$ & $520(64)$ & 0.63 \\
\hline Duration of night sleep (min/day) & $528(53)$ & $521(56)$ & 0.051 \\
\hline General obesity, $n(\%)^{\mathrm{b}}$ & $546(12.0)$ & $38(18.4)$ & 0.006 \\
\hline Central obesity, $n(\%)^{\mathrm{c}}$ & $437(9.6)$ & $33(16.0)$ & 0.003 \\
\hline High body fat, $n(\%)^{\mathrm{d}}$ & $359(7.9)$ & $25(12.1)$ & 0.030 \\
\hline
\end{tabular}

Data are mean (SD) or number (\%)

${ }^{\mathrm{a}}$ Mean numbers of siblings per participant.

${ }^{\mathrm{b}}$ General obesity was defined as BMI $z$ score $>+2$ SD for age- and sex-specific distribution based on the WHO growth reference

${ }^{\mathrm{c}}$ Central obesity was defined as waist circumference $\geq 90$ th percentile for age- and sex-specific distribution using NHANES III reference

${ }^{\mathrm{d}}$ High body fat was defined as body fat $\geq 90$ th percentile for age- and sex-specific distribution using NHANES IV reference

breastfeeding status, or the country's income classification with the risk of childhood obesity, central obesity and high body fat (all $p$ values for interactions are $>0.05$ ).

\section{Discussion}

In this multinational cross-sectional study, we found that maternal GDM was associated with increased odds of obesity and central obesity in children aged 9-11 years old in 12 countries; however, these associations were not fully independent of maternal BMI.

Early research in the Pima Indian Study demonstrated that the offspring of Pima Indian women with diabetes prior to pregnancy and GDM were heavier at birth and had much higher rates of obesity at age 5-19 years than the offspring of prediabetic or non-diabetic women $[5,26]$. In the Northwestern University Diabetes in Pregnancy Study, diabetes during pregnancy, including both GDM and insulin-treated diabetes prior to pregnancy, was associated with increased BMI of the offspring 
Table 3 Mean $z$ scores for BMI, waist circumference and percentage body fat among offspring by maternal GDM status

\begin{tabular}{|c|c|c|c|c|c|c|c|c|c|}
\hline \multirow[t]{2}{*}{ Outcome } & \multicolumn{3}{|l|}{ BMI $z$ score } & \multicolumn{3}{|c|}{ Waist circumference $z$ score } & \multicolumn{3}{|c|}{ Body fat $z$ score } \\
\hline & Non-GDM & GDM & $p$ value & Non-GDM & GDM & $p$ value & Non-GDM & GDM & $p$ value \\
\hline No. of participants & 4534 & 206 & & 4534 & 206 & & 5434 & 206 & \\
\hline Model $1^{\mathrm{a}}$ & $0.48(0.08)$ & $0.70(0.12)$ & 0.012 & $-0.04(0.03)$ & $0.06(0.05)$ & 0.006 & $-0.05(0.09)$ & $0.13(0.12)$ & 0.01 \\
\hline Model $2^{\mathrm{b}}$ & $0.54(0.08)$ & $0.75(0.11)$ & 0.010 & $-0.03(0.03)$ & $0.07(0.04)$ & 0.006 & $0.02(0.09)$ & $0.20(0.11)$ & 0.009 \\
\hline Model $3^{\mathrm{c}}$ & $0.54(0.08)$ & $0.71(0.11)$ & 0.045 & $-0.02(0.03)$ & $0.06(0.04)$ & 0.021 & $0.02(0.09)$ & $0.17(0.11)$ & 0.027 \\
\hline Model $4^{\mathrm{d}}$ & $0.51(0.08)$ & $0.60(0.11)$ & 0.29 & $-0.04(0.03)$ & $0.01(0.05)$ & 0.14 & $-0.01(0.09)$ & $0.10(0.11)$ & 0.13 \\
\hline
\end{tabular}

Data are mean (SE)

${ }^{a}$ Model 1 adjusted for child sex

${ }^{\mathrm{b}}$ Model 2 adjusted for maternal age at delivery and education, infant feeding mode, gestational age, number of younger siblings, child unhealthy diet pattern scores, moderate-to-vigorous physical activity, sleeping time, sedentary time and sex

${ }^{\mathrm{c}}$ Model 3 adjusted for variables in model 2 and birthweight

${ }^{\mathrm{d}}$ Model 4 adjusted for variables in model 3 and current maternal BMI

at birth and after 5 years of age [27]. However, other studies failed to find a clear association between maternal GDM and obesity in children aged 5 years or older [7-11]. One study found that prenatal exposure to the metabolic effects of mild, diet-treated GDM did not increase the risk of childhood obesity [7]. Another study also found little association between maternal glucose during pregnancy and obesity in offspring at 2 years [10]. A systematic review of 12 studies reported that the crude

Table 4 Odds ratios of childhood obesity by maternal GDM status at all or at different BMI levels

\begin{tabular}{|c|c|c|c|c|c|c|c|c|c|}
\hline \multirow[t]{2}{*}{ Outcome } & \multicolumn{3}{|c|}{ General obesity } & \multicolumn{3}{|c|}{ Central obesity } & \multicolumn{3}{|c|}{ High body fat } \\
\hline & Non-GDM & GDM & $p$ value & Non-GDM & GDM & $p$ value & Non-GDM & GDM & $p$ value \\
\hline \multicolumn{10}{|l|}{ Total maternal sample } \\
\hline No. of participants & 4534 & 206 & & 4534 & 206 & & 5434 & 206 & \\
\hline No. of cases & 546 & 38 & & 437 & 33 & & 359 & 25 & \\
\hline Model $1^{\mathrm{a}}$ & 1.00 & $1.65(1.13,2.41)$ & 0.009 & 1.00 & $1.83(1.23,2.72)$ & 0.003 & 1.00 & $1.55(0.99,2.42)$ & 0.06 \\
\hline Model $2^{\mathrm{b}}$ & 1.00 & $1.62(1.10,2.40)$ & 0.015 & 1.00 & $1.83(1.21,2.77)$ & 0.004 & 1.00 & $1.51(0.96,2.40)$ & 0.08 \\
\hline Model $3^{c}$ & 1.00 & $1.53(1.03,2.27)$ & 0.034 & 1.00 & $1.73(1.14,2.62)$ & 0.010 & 1.00 & $1.42(0.90,2.26)$ & 0.14 \\
\hline Model $4^{\mathrm{d}}$ & 1.00 & $1.37(0.92,2.04)$ & 0.13 & 1.00 & $1.54(1.01,2.35)$ & 0.046 & 1.00 & $1.30(0.81,2.06)$ & 0.29 \\
\hline \multicolumn{10}{|l|}{ Maternal overweight } \\
\hline No. of participants & 2083 & 130 & & 2083 & 130 & & 2083 & 130 & \\
\hline No. of cases & 338 & 34 & & 284 & 28 & & 251 & 23 & \\
\hline Model $1^{\mathrm{a}}$ & 1.00 & $1.77(1.16,2.70)$ & 0.009 & 1.00 & $1.71(1.09,2.68)$ & 0.019 & 1.00 & $1.54(0.95,2.51)$ & 0.08 \\
\hline Model $2^{\mathrm{b}}$ & 1.00 & $1.75(1.13,2.73)$ & 0.013 & 1.00 & $1.76(1.10,2.83)$ & 0.019 & 1.00 & $1.53(0.92,2.55)$ & 0.10 \\
\hline Model $3^{\mathrm{c}}$ & 1.00 & $1.60(1.02,2.51)$ & 0.04 & 1.00 & $1.62(1.00,2.61)$ & 0.048 & 1.00 & $1.42(0.85,2.39)$ & 0.18 \\
\hline \multicolumn{10}{|l|}{ Maternal normal weight } \\
\hline No. of participants & 2451 & 76 & & 2451 & 76 & & 2451 & 76 & \\
\hline No. of cases & 208 & 4 & & 153 & 5 & & 108 & 2 & \\
\hline Model $1^{\mathrm{a}}$ & 1.00 & $0.62(0.22,1.76)$ & 0.37 & 1.00 & $1.22(0.47,3.15)$ & 0.69 & 1.00 & $0.58(0.14,2.43)$ & 0.46 \\
\hline Model $2^{\mathrm{b}}$ & 1.00 & $0.63(0.22,1.81)$ & 0.39 & 1.00 & $1.26(0.48,3.34)$ & 0.64 & 1.00 & $0.59(0.14,2.50)$ & 0.47 \\
\hline Model $3^{\mathrm{c}}$ & 1.00 & $0.64(0.22,1.83)$ & 0.40 & 1.00 & $1.27(0.48,3.35)$ & 0.63 & 1.00 & $0.59(0.14,2.49)$ & 0.47 \\
\hline
\end{tabular}

${ }^{\mathrm{a}}$ Model 1 adjusted for child age and sex

${ }^{\mathrm{b}}$ Model 2 adjusted for maternal age at delivery and education, infant feeding mode, gestational age, number of younger siblings, child unhealthy diet pattern scores, moderate-to-vigorous physical activity, sleeping time, sedentary time, age and sex

${ }^{\mathrm{c}}$ Model 3 adjusted for variables in model 2 and birthweight

${ }^{\mathrm{d}}$ Model 4 adjusted for variables in model 3 and current maternal BMI 
Table 5 Odds ratios of childhood obesity by maternal GDM status of various subgroups

\begin{tabular}{|c|c|c|c|c|c|c|c|c|c|}
\hline & \multicolumn{3}{|c|}{ General obesity } & \multicolumn{3}{|c|}{ Central obesity } & \multicolumn{3}{|c|}{ High body fat } \\
\hline & Non-GDM & GDM & $p$ value & Non-GDM & GDM & $p$ value & Non-GDM & GDM & $p$ value \\
\hline \multicolumn{10}{|l|}{ Sex of child ${ }^{\mathrm{a}}$} \\
\hline \multicolumn{10}{|l|}{ Boys } \\
\hline No. of participants & 2091 & 95 & & 2091 & 95 & & 2091 & 95 & \\
\hline No. of cases & 323 & 19 & & 236 & 13 & & 126 & 7 & \\
\hline Model $1^{\mathrm{b}}$ & 1.00 & $1.31(0.74,2.30)$ & 0.35 & 1.00 & $1.26(0.65,2.43)$ & 0.50 & 1.00 & $1.21(0.52,2.82)$ & 0.67 \\
\hline Model $2^{\mathrm{c}}$ & 1.00 & $1.26(0.72-2.19)$ & 0.42 & 1.00 & $1.17(0.61,2.24)$ & 0.64 & 1.00 & $1.15(0.49,2.67)$ & 0.75 \\
\hline \multicolumn{10}{|l|}{ Girls } \\
\hline No. of participants & 2443 & 111 & & 2443 & 111 & & 2443 & 111 & \\
\hline No. of cases & 223 & 19 & & 201 & 20 & & 233 & 18 & \\
\hline Model $1^{\mathrm{b}}$ & 1.00 & $1.72(0.99,2.99)$ & 0.055 & 1.00 & $2.06(1.19,3.56)$ & 0.01 & 1.00 & $1.50(0.85,2.63)$ & 0.16 \\
\hline Model $2^{\mathrm{c}}$ & 1.00 & $1.53(0.88,2.68)$ & 0.13 & 1.00 & $1.81(1.05,3.13)$ & 0.033 & 1.00 & $1.35(0.77,2.37)$ & 0.30 \\
\hline \multicolumn{10}{|l|}{ GDM diagnostic criteria $^{a}$} \\
\hline \multicolumn{10}{|l|}{ ADA criteria } \\
\hline No. of participants & 2328 & 125 & & 2328 & 125 & & 2328 & 125 & \\
\hline No. of cases & 258 & 23 & & 199 & 21 & & 190 & 14 & \\
\hline Model $1^{\mathrm{b}}$ & 1.00 & $1.41(0.85,2.32)$ & 0.18 & 1.00 & $1.77(1.04,2.99)$ & 0.034 & 1.00 & $1.09(0.59,2.01)$ & 0.79 \\
\hline Model $2^{\mathrm{c}}$ & 1.00 & $1.50(0.91,2.48)$ & 0.11 & 1.00 & $1.91(1.12,3.24)$ & 0.017 & 1.00 & $1.23(0.67,2.27)$ & 0.51 \\
\hline \multicolumn{10}{|l|}{ WHO criteria } \\
\hline No. of participants & 2206 & 81 & & 2206 & 81 & & 2206 & 81 & \\
\hline No. of cases & 288 & 15 & & 238 & 12 & & 169 & 11 & \\
\hline Model $1^{\mathrm{b}}$ & 1.00 & $1.76(0.91,3.38)$ & 0.09 & 1.00 & $1.76(0.88,3.52)$ & 0.11 & 1.00 & $2.24(1.08,4.64)$ & 0.03 \\
\hline Model $2^{\mathrm{c}}$ & 1.00 & $1.23(0.64,2.36)$ & 0.53 & 1.00 & $1.11(0.61,2.40)$ & 0.60 & 1.00 & $1.52(0.73,3.14)$ & 0.26 \\
\hline \multicolumn{10}{|c|}{ Moderate-to-vigorous physical activity ${ }^{\mathrm{a}}$} \\
\hline \multicolumn{10}{|l|}{ High level } \\
\hline No. of participants & 2273 & 101 & & 2273 & 101 & & 2273 & 101 & \\
\hline No. of cases & 179 & 13 & & 124 & 10 & & 85 & 6 & \\
\hline Model $1^{\mathrm{b}}$ & 1.00 & $1.57(0.82,3.01)$ & 0.18 & 1.00 & $1.91(0.93,3.92)$ & 0.08 & 1.00 & $1.44(0.58,3.60)$ & 0.44 \\
\hline Model $2^{\mathrm{c}}$ & 1.00 & $1.29(0.65,2.53)$ & 0.47 & 1.00 & $1.52(0.72,3.21)$ & 0.019 & 1.00 & $1.06(0.41,2.78)$ & 0.90 \\
\hline \multicolumn{10}{|l|}{ Low level } \\
\hline No. of participants & 2261 & 105 & & 2261 & 105 & & 2261 & 105 & \\
\hline No. of cases & 367 & 25 & & 313 & 23 & & 274 & 19 & \\
\hline Model $1^{\mathrm{b}}$ & 1.00 & $1.52(0.93,2.48)$ & 0.10 & 1.00 & $1.68(1.02,2.77)$ & 0.044 & 1.00 & $1.43(0.84,2.43)$ & 0.19 \\
\hline Model $2^{\mathrm{c}}$ & 1.00 & $1.40(0.85,2.31)$ & 0.19 & 1.00 & $1.54(0.92,2.59)$ & 0.10 & 1.00 & $1.34(0.78,2.29)$ & 0.29 \\
\hline \multicolumn{10}{|l|}{ Unhealthy diet scores ${ }^{\text {a }}$} \\
\hline High level & & & & & & & & & \\
\hline No. of participants & 2267 & 103 & & 2267 & 103 & & 2267 & 103 & \\
\hline No. of cases & 270 & 21 & & 210 & 17 & & 179 & 11 & \\
\hline Model $1^{\mathrm{b}}$ & 1.00 & $1.67(0.97,2.88)$ & 0.07 & 1.00 & $1.84(1.03,3.29)$ & 0.041 & 1.00 & $1.78(0.59,2.34)$ & 0.65 \\
\hline Model $2^{\mathrm{c}}$ & 1.00 & $1.51(0.87,2.62)$ & 0.14 & 1.00 & $1.69(0.93,3.05)$ & 0.08 & 1.00 & $1.03(0.51,2.07)$ & 0.94 \\
\hline Low level & & & & & & & & & \\
\hline No. of participants & 2267 & 103 & & 2267 & 103 & & 2267 & 103 & \\
\hline No. of cases & 276 & 17 & & 227 & 16 & & 180 & 14 & \\
\hline Model $1^{\mathrm{b}}$ & 1.00 & $1.46(0.82,2.60)$ & 0.19 & 1.00 & $1.65(0.90,3.01)$ & 0.10 & 1.00 & $1.81(0.95,3.44)$ & 0.07 \\
\hline Model $2^{\mathrm{c}}$ & 1.00 & $1.29(0.71,2.32)$ & 0.40 & 1.00 & $1.45(0.78,2.70)$ & 0.24 & 1.00 & $1.67(0.87,3.21)$ & 0.12 \\
\hline Sleep time $\mathrm{e}^{\mathrm{a}}$ & & & & & & & & & \\
\hline High level & & & & & & & & & \\
\hline No. of participants & 2282 & 90 & & 2282 & 90 & & 2282 & 90 & \\
\hline No. of cases & 207 & 13 & & 170 & 12 & & 147 & 8 & \\
\hline Model $1^{\mathrm{b}}$ & 1.00 & $1.72(0.90,3.29)$ & 0.10 & 1.00 & $2.04(1.04,4.02)$ & 0.039 & 1.00 & $1.41(0.64,3.14)$ & 0.40 \\
\hline Model $2^{\mathrm{c}}$ & 1.00 & $1.56(0.80,2.98)$ & 0.20 & 1.00 & $1.83(0.92,3.63)$ & 0.08 & 1.00 & $1.28(0.58,2.84)$ & 0.54 \\
\hline Low level & & & & & & & & & \\
\hline No. of participants & 2252 & 116 & & 2252 & 116 & & 2252 & 116 & \\
\hline No. of cases & 339 & 25 & & 267 & 21 & & 212 & 17 & \\
\hline Model $1^{\mathrm{b}}$ & 1.00 & $1.55(0.94,2.54)$ & 0.08 & 1.00 & $1.69(0.99,2.88)$ & 0.054 & 1.00 & $1.59(0.89,2.82)$ & 0.12 \\
\hline Model $2^{\mathrm{c}}$ & 1.00 & $1.39(0.84,2.30)$ & 0.21 & 1.00 & $1.52(0.88,2.63)$ & 0.13 & 1.00 & $1.45(0.81,2.60)$ & 0.22 \\
\hline Region $^{\mathrm{a}}$ & & & & & & & & & \\
\hline High income countries & & & & & & & & & \\
\hline No. of participants & 2320 & 130 & & 2320 & 130 & & 2320 & 130 & \\
\hline No. of cases & 255 & 20 & & 175 & 20 & & 166 & 15 & \\
\hline Model $1^{\mathrm{b}}$ & 1.00 & $1.23(0.72,2.08)$ & 0.44 & 1.00 & $1.85(1.08,3.18)$ & 0.023 & 1.00 & $1.37(0.74,2.53)$ & 0.32 \\
\hline Model $2^{\mathrm{c}}$ & 1.00 & $1.14(0.67,1.93)$ & 0.64 & 1.00 & $1.70(0.98,2.95)$ & 0.059 & 1.00 & $1.28(0.69,2.38)$ & 0.43 \\
\hline
\end{tabular}


Table 5 (continued)

\begin{tabular}{|c|c|c|c|c|c|c|c|c|c|}
\hline & \multicolumn{3}{|c|}{ General obesity } & \multicolumn{3}{|c|}{ Central obesity } & \multicolumn{3}{|c|}{ High body fat } \\
\hline & Non-GDM & GDM & $p$ value & Non-GDM & GDM & $p$ value & Non-GDM & GDM & $p$ value \\
\hline \multicolumn{10}{|c|}{ Low-middle income countries } \\
\hline No. of participants & 2214 & 76 & & 2214 & 76 & & 2214 & 76 & \\
\hline No. of cases & 291 & 18 & & 262 & 13 & & 193 & 10 & \\
\hline Model $1^{\mathrm{b}}$ & 1.00 & $1.93(1.03,3.62)$ & 0.041 & 1.00 & $1.38(0.70,2.71)$ & 0.36 & 1.00 & $1.43(0.69,3.00)$ & 0.34 \\
\hline Model $2^{\mathrm{c}}$ & 1.00 & $1.66(0.87,3.16)$ & 0.13 & 1.00 & $1.19(0.60,2.38)$ & 0.62 & 1.00 & $1.23(0.58,2.59)$ & 0.60 \\
\hline \multicolumn{10}{|l|}{ Infant feeding mode ${ }^{a}$} \\
\hline \multicolumn{10}{|l|}{ Exclusive breast feeding } \\
\hline No. of participants & 1722 & 63 & & 1722 & 63 & & 1722 & 63 & \\
\hline No. of cases & 190 & 14 & & 161 & 12 & & 110 & 10 & \\
\hline Model $1^{\mathrm{b}}$ & 1.00 & $2.12(1.09,4.14)$ & 0.028 & 1.00 & $2.20(1.09,4.45)$ & 0.028 & 1.00 & $2.34(1.10,4.98)$ & 0.03 \\
\hline Model $2^{\mathrm{c}}$ & 1.00 & $1.57(0.78,3.14)$ & 0.21 & 1.00 & $1.64(0.79,3.40)$ & 0.19 & 1.00 & $1.69(0.77,3.72)$ & 0.19 \\
\hline \multicolumn{10}{|c|}{ Not exclusive breast feeding } \\
\hline No. of participants & 2812 & 143 & & 2812 & 143 & & 2812 & 143 & \\
\hline No. of cases & 356 & 24 & & 276 & 21 & & 249 & 15 & \\
\hline Model $1^{\mathrm{b}}$ & 1.00 & $1.44(0.89,2.34)$ & 0.14 & 1.00 & $1.66(0.99,2.78)$ & 0.054 & 1.00 & $1.20(0.67,2.15)$ & 0.55 \\
\hline Model $2^{\mathrm{c}}$ & 1.00 & $1.37(0.84,2.25)$ & 0.21 & 1.00 & $1.24(0.95,2.71)$ & 0.08 & 1.00 & $1.16(0.65,2.10)$ & 0.62 \\
\hline
\end{tabular}

${ }^{\text {a }}$ All $p$ values for interactions are $>0.05$.

${ }^{\mathrm{b}}$ Model 1 adjusted for maternal age at delivery and education, infant feeding mode, gestational age, number of younger siblings, child unhealthy diet pattern scores, moderate-to-vigorous physical activity, sleeping time, sedentary time, sex and birthweight, other than the variable for stratification

${ }^{\mathrm{c}}$ Model 2 adjusted for variables in model 1 and current maternal BMI

odds ratios for the relationship between maternal GDM and childhood overweight and obesity ranged from 0.7 to 6.3 and the association was not statistically significant in eight of the studies [28]. Most of these studies were conducted in high income countries, with only one from a middle income country [29]. Therefore, large studies using uniform methods to assess maternal GDM and childhood obesity across various populations are needed to evaluate this question. Our study is the first to evaluate the association between maternal GDM and childhood obesity using such widespread, multinational data. We found that maternal GDM was associated with an increased risk of childhood obesity among children aged 9-11 years from 12 countries. Moreover, our results indicate that the positive associations between maternal GDM and the risk of childhood obesity were significant among children from low to middle income countries and between maternal GDM and an increased risk of central obesity among children from high income countries; however, these associations were no longer significant after additional adjustment for current maternal BMI (Table 5).

Several prenatal and perinatal factors including maternal prepregnancy BMI, gestational weight gain, maternal GDM and child birthweight have been found to be associated with an increased risk of obesity in offspring [2, 30,31]. Previous studies have demonstrated the significant association between maternal GDM and an increased risk of childhood obesity to be both independent of [32] and dependent on [33] maternal prepregnancy BMI. Since maternal prepregnancy obesity is a risk factor for maternal GDM [34], adjustment for maternal prepregnancy BMI as a confounding factor or as a proxy for genetic predisposition in the analysis of maternal GDM and childhood obesity may be over-adjusted. The present study found that the positive association between maternal GDM and childhood obesity risk was only slightly attenuated by birthweight and was not fully independent of current maternal BMI. We used current maternal BMI (postpartum BMI at about 10 years) but not maternal prepregnancy BMI in the multivariable-model for three reasons: first, data on maternal prepregnancy BMI and gestational weight gain were not available in the present study; second, a strong correlation (0.827) has been shown between maternal prepregnancy BMI and current BMI [35]; third, although current maternal BMI represents shared postnatal environmental factors, we still included current maternal BMI in the final multivariable-adjusted model (Model 4) which can partially control for the maternal prepregnancy BMI effect.

Inconsistent associations have been found in studies that assessed children at different ages. The Northwestern University Diabetes in Pregnancy Study reported that the relative weight in children of GDM mothers increased dramatically after 5 years of age, and that half of the children of GDM mothers had a weight $>90$ th percentile by age 8 years [6]. One study in China found that maternal GDM increased the cardiometabolic risk in early childhood at 8 years of age, but not at 15 years of age [36]. Our study found that the children of GDM mothers had 1.42-1.73 times higher odds of developing obesity than children of non-GDM mothers at 9-11 years old. A prospective pregnancy cohort from the UK reported that the odds ratio of obesity among children of GDM mothers at 9- 
11 years old was $1.51(0.76,2.98)$, which is similar to our study [37].

Waist circumference, BMI and body fat are the three main indicators used to evaluate obesity. In the USA, the National Institutes of Health clinical guidelines for the identification and treatment of overweight and obesity among adults recognise the importance of including measurements of both obesity and central obesity, which are assessed by BMI and waist circumference, respectively [38]. Some studies have established that central obesity predicts obesity-related health risk [39]. However, the majority of the available studies used BMI, and few studies have included concurrent measurements of waist circumference and body fat [11, 37, 40]. Our study demonstrated a positive association between maternal GDM and the odds of childhood obesity and central obesity; however, after additional adjustment for current maternal BMI, this association was only significant for central obesity and not for general obesity. Therefore, more studies are needed to confirm the effect of maternal GDM on the risks of childhood obesity and central obesity.

The mechanisms by which exposure to diabetes in utero increases the risk of offspring obesity are not fully understood. Exposure to maternal diabetes is associated with excess fetal growth in utero, possibly mainly due to an increase in fetal fat mass and alterations in fetal hormone levels [2]. In addition, exposure to maternal diabetes results in elevated hyperglycaemia hyperinsulinaemia and elevated leptin synthesis in offspring [2]. Maternal prenatal GDM may also influence the fetal epigenome; thereby influencing the expression of genes that direct the accumulation of body fat or related metabolism [2].

There were several strengths of our study including the recruitment of a large multinational sample of children from low to high income countries across several regions of the world, the highly standardised measurement protocol, the use of direct measurements whenever possible and the rigorous quality control programme. In addition, body weight, waist circumference and body fat were directly measured by standardised methods. One limitation of the study is that it is a cross-sectional study. Thus, we could not make cause-andeffect inferences. Second, since data on maternal prepregnancy BMI and gestational weight gain were not available, we were not able to assess the effect of these variables on the association of GDM with the risk of childhood obesity. Third, the information on maternal GDM status, current maternal body weight and height, infant feeding mode, gestational age and child's birthweight was recalled by parents in a selfreported questionnaire, which may have introduced recall bias. Although no specific assessment of validity of selfreporting of these variables was carried out in the present study, similar questionnaires have been used in a large number of epidemiological studies. Finally, maternal GDM was diagnosed by different criteria across the different sites, which may bias the results.
In conclusion, GDM was associated with an increased risk of obesity among children aged 9-11 years; however, this association was not fully independent of maternal BMI. Furthermore, this study provided evidence for a long-term effect of the different diagnostic criteria for maternal GDM on the risk of childhood obesity.

Acknowledgements We wish to thank the ISCOLE External Advisory Board and the ISCOLE participants and their families who made this study possible. A list of members of the ISCOLE Research Group is given in the electronic supplementary material (ESM).

Funding ISCOLE was funded by The Coca-Cola Company. GH was supported by a grant from the National Institute of Diabetes and Digestive and Kidney Diseases of the National Institutes of Health under Award Number R01DK100790. The funder had no role in the design and conduct of the study, the collection, management, analysis and interpretation of the data, or preparation, review and approval of the manuscript.

Duality of interest MF has received a research grant from Fazer Finland and has received an honorarium for speaking for Merck. AK has been a member of the Advisory Boards of Dupont and McCain Foods. RK has received a research grant from Abbott Nutrition Research and Development. VM is a member of the Scientific Advisory Board of Actigraph and has received an honorarium for speaking for The Coca-Cola Company. TO has received an honorarium for speaking for The Coca-Cola Company. The authors report no other potential conflicts of interest.

Contribution statement $\mathrm{PZ}$ and YQ designed the study, acquired the data, performed the statistical analysis, interpreted the data, drafted the article and approved the final version to be published. GH designed the study, acquired the data, suggested some reanalyses, reviewed and critically revised the article and approved the final version to be published. EL, PTK, J-PC, MF, WDJ, RK, AK, EVL, CM, JARM, VM, TO, VO, OLS, MS, MST and CT-L acquired the data, reviewed and critically revised the article and approved the final version to be published. GH is responsible for the integrity of the work as a whole.

\section{References}

1. Wu JF (2013) Childhood obesity: a growing global health hazard extending to adulthood. Pediatr Neonatol 54:71-72

2. Dabelea D (2007) The predisposition to obesity and diabetes in offspring of diabetic mothers. Diabetes Care 30:S169-S174

3. World Health Organization (1999) Definition, diagnosis and classification of diabetes mellitus and its complications. World Health Organization, Geneva

4. Jiwani A, Marseille E, Lohse N, Damm P, Hod M, Kahn JG (2012) Gestational diabetes mellitus: results from a survey of country prevalence and practices. J Matern Fetal Neonatal Med 25:600-610

5. Pettitt DJ, Nelson RG, Saad MF, Bennett PH, Knowler WC (1993) Diabetes and obesity in the offspring of Pima Indian women with diabetes during pregnancy. Diabetes Care 16:310-314

6. Silverman BL, Rizzo T, Green OC et al (1991) Long-term prospective evaluation of offspring of diabetic mothers. Diabetes 40:121-125

7. Whitaker RC, Pepe MS, Seidel KD, Wright JA, Knopp RH (1998) Gestational diabetes and the risk of offspring obesity. Pediatrics 101:E9 
8. Gillman MW, Rifas-Shiman S, Berkey CS, Field AE, Colditz GA (2003) Maternal gestational diabetes, birth weight, and adolescent obesity. Pediatrics 111:e221-e226

9. Tam WH, Ma RC, Yang X et al (2008) Glucose intolerance and cardiometabolic risk in children exposed to maternal gestational diabetes mellitus in utero. Pediatrics 122:1229-1234

10. Pettitt DJ, McKenna S, McLaughlin C, Patterson CC, Hadden DR, McCance DR (2010) Maternal glucose at 28 weeks of gestation is not associated with obesity in 2-year-old offspring: The Belfast Hyperglycemia and Adverse Pregnancy Outcome (HAPO) Family Study. Diabetes Care 33:1219-1223

11. Patel S, Fraser A, Davey Smith G et al (2012) Associations of gestational diabetes, existing diabetes, and glycosuria with offspring obesity and cardiometabolic outcomes. Diabetes Care 35:63-71

12. Regnault N, Gillman MW, Rifas-Shiman SL, Eggleston E, Oken E (2013) Sex-specific associations of gestational glucose tolerance with childhood body composition. Diabetes Care 36:3045-3053

13. Klein S, Allison DB, Heymsfield SB et al (2007) Waist circumference and cardiometabolic risk: a consensus statement from Shaping America's health: Association for Weight Management and Obesity Prevention; NAASO, the Obesity Society; the American Society for Nutrition; and the American Diabetes Association. Obesity 15: 1061-1067

14. Katzmarzyk PT, Barreira TV, Broyles ST et al (2013) The International Study of Childhood Obesity, Lifestyle and the Environment (ISCOLE): design and methods. BMC Public Health 13:900

15. Alberti KG, Zimmet PZ (1998) Definition, diagnosis and classification of diabetes mellitus and its complications. Part 1: diagnosis and classification of diabetes mellitus provisional report of a WHO consultation. Diabet Med 15:539-553

16. American Diabetes Association (2000) Gestational diabetes mellitus. Diabetes Care 23:S77

17. Currie C, Gabhainn SN, Godeau E et al. (eds) (2008) Inequalities in children's health: HBSC international report from the 2005/2006 survey. World Health Organization, Geneva

18. Mikkilä V, Vepsäläinen H, Saloheimo T et al (2015) An international comparison of dietary patterns in 9-11-year-old children. Int J Obes Suppl 5:S17-S21

19. Saloheimo T, González SA, Erkkola M et al (2015) The reliability and validity of a short food frequency questionnaire among 9-11year olds: a multinational study on three middle-income and highincome countries. Int J Obes Suppl 5:S22-S28

20. McDowell MA, Fryar CD, Ogden CL (2009) Anthropometric reference data for children and adults: United States, 1988-1994. Vital and health statistics Series 11, Data from the national health survey: 1-68

21. Laurson KR, Eisenmann JC, Welk GJ (2011) Body fat percentile curves for U.S. children and adolescents. Am J Prev Med 41:S87-S92

22. Fernandez JR, Redden DT, Pietrobelli A, Allison DB (2004) Waist circumference percentiles in nationally representative samples of African-American, European-American, and Mexican-American children and adolescents. J Pediatr 145:439-444

23. Barreira TV, Schuna JM Jr, Mire EF et al (2015) Identifying children's nocturnal sleep using 24-h waist accelerometry. Med Sci Sports Exerc 47:937-943

24. Tudor-Locke C, Barreira TV, Schuna JM Jr, Mire EF, Katzmarzyk PT (2014) Fully automated waist-worn accelerometer algorithm for detecting children's sleep-period time separate from 24-h physical activity or sedentary behaviors. Appl Physiol Nutr Metab 39:53-57

25. Evenson KR, Catellier DJ, Gill K, Ondrak KS, McMurray RG (2008) Calibration of two objective measures of physical activity for children. J Sports Sci 26:1557-1565

26. Pettitt DJ, Baird HR, Aleck KA, Bennett PH, Knowler WC (1983) Excessive obesity in offspring of Pima Indian women with diabetes during pregnancy. N Engl J Med 308:242-245

27. Silverman BL, Rizzo TA, Cho NH, Metzger BE (1998) Long-term effects of the intrauterine environment. Northwest Univ Diabetes Pregnancy Center. Diabetes Care 21:B142-B149

28. Kim SY, England JL, Sharma JA, Njoroge T (2011) Gestational diabetes mellitus and risk of childhood overweight and obesity in offspring: a systematic review. Exp Diabetes Res 2011:541308

29. Zhang S, Liu H, Zhang C et al (2015) Maternal glucose during pregnancy and after delivery in women with gestational diabetes mellitus on overweight status of their children. BioMed Res Int 2015:543038

30. Qiao Y, Ma J, Wang Y et al (2015) Birth weight and childhood obesity: a 12-country study. Int J Obes Suppl 5:S74-S79

31. Li N, Liu E, Guo J et al (2013) Maternal prepregnancy body mass index and gestational weight gain on offspring overweight in early infancy. PLoS One 8:e77809

32. Deierlein AL, Siega-Riz AM, Chantala K, Herring AH (2011) The association between maternal glucose concentration and child BMI at age 3 years. Diabetes Care 34:480-484

33. Philipps LH, Santhakumaran S, Gale C et al (2011) The diabetic pregnancy and offspring BMI in childhood: a systematic review and meta-analysis. Diabetologia 54:1957-1966

34. Li N, Liu E, Guo J et al (2013) Maternal prepregnancy body mass index and gestational weight gain on pregnancy outcomes. PLoS One 8:e82310

35. Hu G, Tian H, Zhang F et al (2012) Tianjin Gestational Diabetes Mellitus Prevention Program: study design, methods, and 1-year interim report on the feasibility of lifestyle intervention program. Diabetes Res Clin Pract 98:508-517

36. Tam WH (2012) The long term effect of maternal gestational diabetes to both the mothers and their offspring. The Chinese University of Hong Kong, Hong Kong. Available from http://repository.lib.cuhk.edu.hk/tc/islandora/object/cuhk\%3 A328716/metadata, accessed 1 August 2012

37. Lawlor DA, Fraser A, Lindsay RS et al (2010) Association of existing diabetes, gestational diabetes and glycosuria in pregnancy with macrosomia and offspring body mass index, waist and fat mass in later childhood: findings from a prospective pregnancy cohort. Diabetologia 53:89-97

38. NHLBI Obesity Education Initiative Expert Panel (1998) Clinical guidelines on the identification, evaluation, and treatment of overweight and obesity in adults. National Heart, Lung, and Blood Institute, Bethesda

39. Janssen I, Katzmarzyk PT, Ross R (2004) Waist circumference and not body mass index explains obesity-related health risk. Am J Clin Nutr 79:379-384

40. Crume T, Ogden L, West $\mathrm{N}$ et al (2011) Association of exposure to diabetes in utero with adiposity and fat distribution in a multiethnic population of youth: the Exploring Perinatal Outcomes among Children (EPOCH) Study. Diabetologia 54:87-92 